\title{
Quality assessment of educational outcomes of university students in conditions of the modernization of higher education
}

\author{
S.N. Shirobokov*, and V.V. Lorents \\ Omsk State Pedagogical University, city of Omsk, Russian Federation
}

\begin{abstract}
The article discusses current problems and opportunities for competitiveness assessment of graduate in the context of the requirements of professional standards. The authors present the technology of competitiveness assessment of a future specialist taking into account the following criteria: professional knowledge in a psychological and pedagogical and subject area, the level of communicative culture in organizing interaction with the subjects of education, the level of student motivation in creating an individual educational route and forming longterm professional goals, reflexive skills of students in assessing the results of learning and cognitive activities and monitoring the dynamics of student progress in the educational space. The article material presents the results of empirical and comparative theoretical study of the level of development of educational outcomes and functional literacy of students of Russian and a number of foreign universities. The competitiveness assessment of future specialists was based on the study of the level of development of universal skills "soft skills" on the example of the implementation and design of individual educational routes of students. The authors have formulated conclusions that this process of assessment of scientific and educational achievements of students is associated with trends in humanization and internationalization of higher education, which determines the need to use in the educational process of a modern university open learning systems aimed at organizing the learning of students on individual educational routes, providing academic mobility of students.
\end{abstract}

\section{Introduction}

The global community has reached a point in the development of educational systems where there is often some isolationism in a certain confrontation with the trends of internationalization and the priorities of international and comparative studies of the quality of educational outcomes of students in different countries and regions. This challenge is evident in various issues and problems: assessment of graduates' competencies, recognition of educational documents, development of new areas of international educational audit, state and public accreditation of basic educational programs, forming an individual route, learning of mobile and competitive staff.

\footnotetext{
* Corresponding author: sshirob@yahoo.com
} 
In the countries of Europe and the United States, recent years have explored how learning and teaching in higher education institutions have evolved in the context of changing demands, technological and social developments, and national-level policies and reforms. The learning and upbringing of students has become the primary mission of all universities. It became a central theme in the discussion of views on the future of the global space of higher education. While the first rounds of the Bologna Process focused on structural reforms, increased mobility, collaboration and international fame, today the focus is on teaching and learning content using digital technologies. Their role in forming a competitive and mobile graduate is becoming apparent, but little explored.

In order to propose concrete practical steps and identify trends to overcome this conflict, it is necessary to study the main organizational and pedagogical conditions of modernization of education in the context of digitalization, as well as to assess the role of international studies of education quality assessment and its contribution to the career creating of future graduates of educational organizations.

The study of constructive international experience in this area in many countries and regions of the world remains relevant and meaningful. However, there is also a lag in comprehending in science the accumulated experience of organizing and conducting international study in quality assessment of educational outcomes of students, including functional literacy, both in Russia and in foreign countries. Experts note that if earlier it was correlated with the empirical lack of study of important problems and data for the industry, now it is due to the lack of conceptual study in the new socio-economic conditions and clearly defined methodological support for the definition and implementation of tools to improve the quality of education at all levels.

\section{Materials and methods}

The theoretical basis of the study were the provisions of theoretical and methodological nature relating to the study of the conditions of the organization of training students for pedagogical design and educational activities, the concept of personality-centered education, system-activity and competence-based approach.

The study was based on the methodology of comparative pedagogy, in particular crossnational and intra-national comparisons, statistical data processing were conducted. Studies at the local level was based on the results of international experience and included the following levels: geographic-local level (universities, states, regions, countries), nonlocalized demographic groups of respondents (age, ethnicity), aspects of education and society as a whole (strengths and weaknesses in the development of modern higher education, Federal State Education Standards, the Bologna process, changes in the labor market, curricula, the influence of administrative structures on the organization of the educational process on modular technology). As empirical methods of study, we used the method of questioning, included pedagogical observation, conversation, interviewing, analysis of activity products (projects of individual educational routes), which allowed to recognize the students' readiness for professional and pedagogical design activity and the level of development of universal super subject skills "soft skills".

Significant updating of teaching and learning materials and recommendations, taking into account the modernization and focus of the entire education system on new achievements and results, is inextricably linked to the functional literacy of students and the development of cognitive interest, motivation to organize self-learning and the formation of behavioral strategies in different situations, readiness to live in an era of instability and the ability to solve problems in fast-changing situations in accordance with the skills of the twenty-first century. The development of universal skills contribute to the preparation of 
graduates of educational organizations for adult and independent life, based on the concept of competitiveness and mobility in the international and domestic labor markets.

Higher education in psychology and pedagogy trains a new type of specialist by means of theoretical study and technological approaches. These approaches provide for the formation of professional and pedagogical reflexive thinking, the development of creative potential and readiness for personal-professional and educational self-improvement.

Domestic and foreign scientists note that targeted and continuous professional development of teachers through a system of training, retraining and professional development, which requires a radical update of the content and methods of teaching are also an effective mechanism to improve the quality of education along with the organization of systematic monitoring of educational outcomes of students using modern meters for a comprehensive assessment of personal, subject, super subject results [1].

Particular attention is now being paid to informing the entire professional community and the public on the results and tools of international study on the quality assessment of student achievement in a comparative perspective.

In pedagogical comparativism, there are theories on the close relationship and continuity of the quality assessment of educational outcomes of schoolchildren with the assessment of students' achievements and the requirements of the international labor market for the evaluation of competencies of university graduates.

Important criteria for quality assessment of graduates include: 1) the time it takes a graduate to adapt to the workplace; 2) the number of specialized (related) professions in which the graduate can work, taking into account the qualifications obtained. When solving these practice-oriented tasks of a future specialist, the task of coordinating the content of training and personal development of the future specialist arises; traditional approach for quality assessment of specialist training and the capabilities of the educational services market; the need for a system of effective diagnostic methods for quality assessment of training in higher education institutions and the lack of scientific and methodological support for current and final quality control of training; the requirements of the labor market for a specialist and the readiness of teachers to prepare a competitive graduate specialist.

Current study in the field of pedagogical comparativism, based on the idea of "dialogue of cultures", pays attention to borrowing the best educational practices and positive experience of leading countries and regions of the world, which have all recognized achievements in developing, designing, using technologies of future professions training, have successfully tested methods of evaluation of educational outcomes, intermediate and final control, contributing to the development of all factors of the educational process [2].

When conducting a comparative-pedagogical study of the best practices of another country, the formed system of quality control and evaluation of education in a particular state cannot be fully copied and transferred from one space to another without testing and adapting such positive experience. The conditions for this application are necessary: training of all teachers in modern and effective technologies to assess the quality of competitiveness and mobility of future specialists, the motivation and desire of students in the need for self-assessment, analysis of experiments on the use of individual methods of interactive and activity-based learning.

At the same time, the strategy and tactics of the Russian higher education system should be based not only on the consideration of progressive international achievements in the evaluation of the quality of training of a competitive specialist, but also take into account the richest heritage - the cultural and historical traditions of training specialists in our country and the accumulated social and pedagogical experience. 


\section{Results}

Higher education in psychology and pedagogy, according to Federal State Education Standards, should train specialists of a new type by means of competence and systemactivity approach. In summary, the training of competitive staff involves the formation of professional and pedagogical reflexive and critical thinking, the development of educational and creative potential, as well as the ability to continuous personal growth and professional development.

From this perspective, the training of the future teacher to design activities, for example in the design of the individual educational route of the student, acts as a part of the professional and pedagogical training in general. The individual educational route is a purposefully designed differentiated educational program that provides the student with the position of the subject of choice, development and implementation of the educational program, with the implementation of pedagogical support for his professional selfdetermination of self-realization.

As the results of our study and practice show, the design of an individual educational route has significant potential for the development of personal, professional and educational competencies of a future teacher, contributes to the fomation of universal skills soft skills.

In accordance with the professional standard of the teacher and the requirements of the Federal State Education Standards new generation, teaching activity is effective and of high quality when a teacher has formed functional literacy, the content of which is social adaptation, readiness to carry out their professional duties in a changing environment, the ability to professional growth and effective solution of professional problems in nonstandard situations.

In this case, the development of design skills of the teacher is of paramount importance, because the teacher must be able to professionally design the process of organization and content of educational activities, enriched with modern technologies of training and education, provide ongoing pedagogical support to students according to the developed individual educational routes, to predict, analyze and anticipate the results of the pedagogical process, model innovative educational programs and projects, plan own professional growth and professional development, and recognize own difficulties.

Designing an individual educational route in the process of preparing a future teacher contributes to the development of functional literacy and the formation of professional competencies necessary to carry out pedagogical activities.

The results of the conducted fact-finding experiment, in which 210 students of the Faculty of Foreign Languages and the Mathematics Department of Omsk State Pedagogical University participated, demonstrate a diverse motivational structure in the field of education and students' awareness of the need to acquire the skills of design activity, in particular the design of individual and educational routes (educational growth, selfdevelopment, spiritual enrichment, the acquisition of a profession).

The individual-educational route was considered as a constituent element of the multilevel system of higher pedagogical education. The concept of multilevel higher pedagogical education is focused on providing individual educational services, allowing the student in the process of learning to choose the individual-educational route, in order to obtain the necessary education, and, on its basis, the appropriate level of professional training. The study of the motivational structure of the student in the construction of educational trajectory allowed us to identify the level of independence and activity of the future specialist in self-assessment of educational outcomes and setting personally significant goals in getting higher education, awareness of the level of personal competitiveness and demand in the labor market. 
According to the results of the empirical study, we identified four components of the motivational sphere of students: cognitive component $(58 \%)$ - acquisition of fundamental knowledge; social (18\%) - getting higher education and status in society; socio-cultural $(15 \%)$ - getting a profession and development of professional values; economic (14\%) earning, getting a high-paid position. The motivational sphere made it possible to identify trends and lines of advancement along the individual educational route.

In the motivational component can be traced to the problem of motivational structure of educational and cognitive activity of the student, where the main motive is the motive of knowledge. The experiment with the introduction of an additional elective aimed at the development of design skills in simulated professional situations and tasks, the involvement of students in research design activities, participation in conferences of international and regional level to discuss the best teaching practices, as well as the design and tracking of the implementation of individual educational routes allowed to achieve dynamics in the studied professional competencies.

The content of each individual-educational route was determined taking into account the need to form the functional literacy of the student and the development of key professional competencies, the specificity of the university and the educational needs of the student (motivational sphere).

Based on the modular technology of designing educational programs, the student, together with the teacher-tutor, built an individual educational route, which includes the following set of modules: the basic invariant module (implementation of the requirements of Federal State Education Standards to the minimum educational content), special module (reflecting the specialization of the university and training profile) and humanitarian module (elective courses). The content and structure of the individual educational route of the student are built according to the following equation (Fig. 1.).

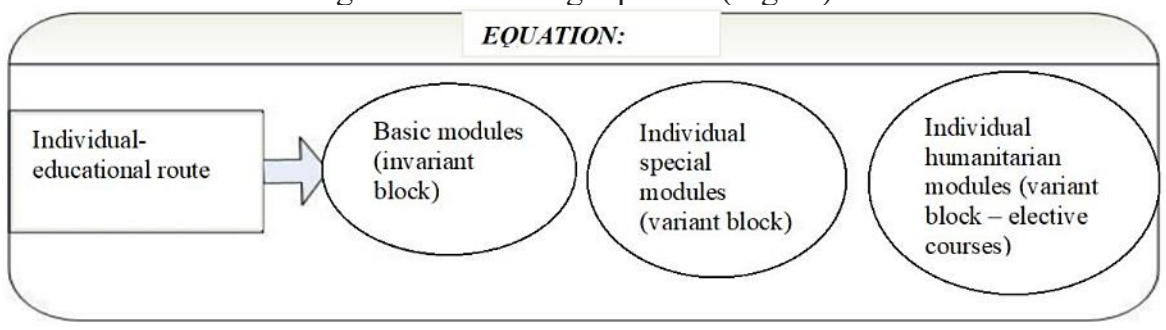

Fig. 1. Equation for determining the structure and content of the individual educational route

Individual educational routes were built according to the following types, according to the motivational sphere: "I-centered", "I am a scientist", "I am a teacher", "gaining knowledge", "educated person".

Individualization of learning was carried out in three stages of the developed and proposed educational route. At the first stage, in the awareness of his role as a learner in the educational environment, there was an awareness of the need for education and the formation of the desire for constant self-realization. The second stage involved full immersion in the educational environment, awareness of the goals and values of education, the orientation on personal self-improvement, actualization and systematization of the acquired knowledge. At this stage, some students with unstable professional orientation had subject differentiation associated with the formation of a selective attitude to the subjects studied. In this case, $5 \%$ of the students changed their study profile.

At the third stage of training in the development of the educational environment was the orientation of the student for continuous personal improvement and awareness of himself in the profession, the vision of himself as a future competitive and mobile 
specialist. Orientation on mastering the profession was transferred in $18 \%$ of students to scientific activity, the desire to continue training in graduate and postgraduate schools was formulated. The dynamics of progress along the individual educational route was tracked in three directions: personal growth, knowledge, and orientation toward professional activity. The logic of assessing the educational outcomes and the construction of long-term and short-term goals on the basis of forecasting was built according to the following scheme (see Fig. 2.)

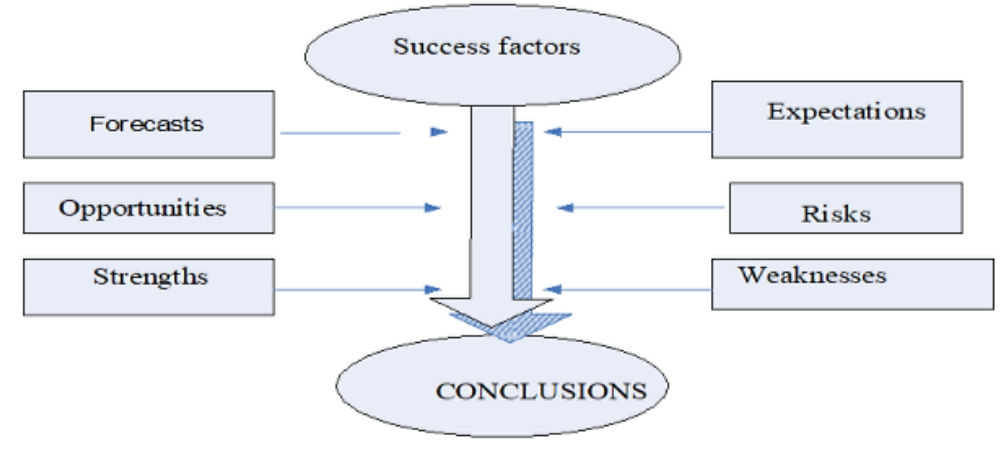

Fig. 2. The logic of predicting personal and educational outcomes.

The criteria of the student's readiness to design an individual educational route is the presence of a personality-oriented task for the implementation of this design activity. Designing the individual educational route of the student is carried out in the process of studying pedagogical disciplines as preparation for design activity and is built step by step, according to the components of readiness for design activity (motivational, cognitive, technological, reflective).

The study also developed methodological guidelines for the implementation of the technology of designing individual educational route, which includes: a) a set of learning situations (assessment and self-assessment, cognitive, design and creative); b) methodological guidelines for the design of individual educational route of the student; c) an individual diary "Profile of my skills" (a method of diagnostic tracking of the results of the design and implementation of the individual educational route of the student); d) monographic characteristics of students.

Thus, the results of the study on the formation of students' abilities to design an individual educational route contributed to determining the quality of training of a specialist in the university, which is assessed by four basic indicators: 1) professional skills (knowledge of the subject area and psychological and pedagogical knowledge); 2) level of communicative culture; 3 ) aspiration for professional growth; 4) ability to reflection.

Organizing a comparative-pedagogical study on the formation of universal soft skills abroad, we paid attention to the relevance of study in the field of development of academic mobility of students [3]. An important aspect of internationalization of higher education and the creation of a single educational space is the academic mobility of students.

If in Russian universities designing an individual educational route and its implementation is more associated with the construction of intra-university trajectory of training, in foreign universities it is an opportunity to develop academic mobility of students.

The study revealed a trend of increasing international competition and partnership between universities, building a flexible, nonlinear educational process focused on academic mobility programs for students and individual learning trajectory [4]. 
The study included 66 students in the graduate programs at the State University of New York at Cortland (USA) and 51 students in the graduate programs at the University of Heidelberg (Germany). The method of questionnaires, conversations and interviews was used to study students' attitudes towards the quality of higher education, the level of competitiveness of future specialists and the requirements of the labor market. The questions were based on self-assessment of professionally significant personal qualities, as well as assessment of educational results and functional literacy of students.

Having studied the study on the motivational sphere of foreign students, we can identify the following aspects in the development of professional self-awareness of students. The majority of American students (65\%) begin thinking about their profession and building their educational route at age $15-20,14 \%$ decide on the direction of further education between the ages of 21 and 25, and the rest of the respondents at age 26, when they enter university.

In Germany, more than half of the respondents-students (55\%) first determine their career direction at age 15-20, i.e. before entering university, $39 \%$ at age $21-25$, i.e. during their studies at university, and $6 \%$ at age $26-30$, when they realize themselves in the profession when receiving higher education and building an educational route [5].

In this regard, awareness of one's competitiveness in the U.S. labor market is "very often" for $11 \%$ of students and "often" for $58 \%$ of students, while $30 \%$ of students rarely think about their professional careers and their relevance in international or domestic labor markets.

In Germany, there is also a tendency for $73 \%$ of students to be self-conscious about their profession, and 27\% rarely think about this question [6].

Studying the level of development of super subject, universal skills and soft skills when performing design activity and goal setting, solving simulated professional tasks of reflective nature, American students (77\%), German students (73\%) and Russian students (75\%) emphasized the need to develop skills of goal setting activity in rapidly changing conditions, application of knowledge in non-standard situations when solving professional learning situations. A competitive specialist, according to respondents, is a specialist who is capable of achieving a goal in an unstable world, ready to perform any task by applying effective study methods, and this one is a specialist with extensive knowledge based on his personal experience.

$68 \%$ of foreign students and $62 \%$ of Russian students noted that a competitive specialist must adapt quickly to new situations and apply innovative technologies, must be able to overcome difficulties, be able to learn throughout life and constantly improve their skills to be in demand in the labor market. On average, about $30 \%$ of respondents pay attention to the development of individual abilities and personality traits that make a specialist unique and in-demand in the profession.

Statistics show that the majority of American and German students have a conscious approach to their choice of profession, and they purposefully design and implement an individual educational route.

Since foreign universities provide the opportunity to study on flexible, non-linear educational programs, promote academic mobility, the level of individualization of higher education is quite high [7].

U.S. universities, for example, have a flexible system for changing specializations and learning profiles. As a result, the vast majority of both American (63\%) and German students $(66 \%)$ are satisfied with the education that they receive [8]. 


\section{Conclusions}

As a result of empirical and comparative theoretical study, the following provisions were formulated:

1. The analysis of the quality assessment of graduates' training in the context of the requirements of the professional standard shows: the evaluation process is a complex structure and content of the procedure, is an integral part of a holistic pedagogical process, and is subject to its general regularities.

2. Designing technologies of competitiveness assessment involves justification and selection of appropriate assessment criteria that are adequate to the set goal. The quality of training of a specialist in the university is evaluated according to four most important criteria: 1) professional skills (knowledge of the subject area and psychological and pedagogical knowledge); 2) level of communicative culture; 3) aspiration for professional growth; 4) ability to reflection.

3. Universal skills "soft skills" should be considered as meaningful benchmarks in Bachelor's and Master's degree programs. In creating conditions for active design activity and involvement of students in innovative processes, subjective position and personal professional self-awareness are developed, and professional growth strategy is built. The development of creative independent cognitive activity, professional and personal stability contribute to the formation of skills of self-development and professional self-expression, the development of critical thinking and the ability to solve professional problems in an era of change.

4. A steady trend of humanization and internationalization of higher education can include the introduction of open learning systems in educational activities, allowing students to customize training according to individual educational routes, which ensure academic mobility of students and their competitiveness.

\section{References}

1. A.I. Adamski, M.V. Boguslavsky, Innovative educational content, 20034.9

2. V.I. Baydenko, Bologna process: structural reform of European higher education, 128 (2003)

3. I.K. Tsalikova, S.V. Pakhotina, Education and Science, 21(8), 187 (2019)

4. J. Bacca, S. Baldiris, R. Fabregat, S. Graf, Kinshuk, Educational Technology and Society, 17(4), 133 (2014)

5. P.G. Altbach, J. Knight, Journal of Studies in International Education, 11(3-4), 290 (2017)

6. E. Baran, Educational Technology and Society, 17(4), 17 (2014)

7. A. Kirkwood, L. Price, Learning, Media and Technology, 39(1), 6 (2014)

8. M. Scheffel, H. Scheffel, S. Stoyanov, Educational Technology and Society, 17(4), 117 (2014) 